\title{
2-GROUPS WITH FEW CONJUGACY CLASSES
}

\author{
NIGEL BOSTON ${ }^{1}$ AND JUDY L. WALKER ${ }^{2}$ \\ ${ }^{1}$ Department of Mathematics, University of Illinois, Urbana, \\ IL 61801, USA (boston@math.uiuc.edu) \\ ${ }^{2}$ Department of Mathematics and Statistics, University of Nebraska, \\ Lincoln, NB 68588, USA (jwalker@math.unl.edu)
}

(Received 3 September 1998)

\begin{abstract}
An old question of Brauer that asks how fast numbers of conjugacy classes grow is investigated by considering the least number $c_{n}$ of conjugacy classes in a group of order $2^{n}$. The numbers $c_{n}$ are computed for $n \leqslant 14$ and a lower bound is given for $c_{15}$. It is observed that $c_{n}$ grows very slowly except for occasional large jumps corresponding to an increase in coclass of the minimal groups $G_{n}$. Restricting to groups that are 2-generated or have coclass at most 3 allows us to extend these computations.
\end{abstract}

Keywords: $p$-groups; conjugacy classes; coclass

AMS 1991 Mathematics subject classification: Primary 20D15

Secondary 20-04; 20D60

\section{Introduction}

There is a long history to the question of the possible number $k(G)$ of conjugacy classes of a finite group $G$. It began in 1903 when Landau [8] showed that only finitely many groups $G$ have a given $k(G)$. This was made explicit in 1963 by Brauer [3] (see also [4]), who showed that $k(G)>\log _{2} \log _{2}|G|$. In general, $k(G)$ will be much larger than this. For example, Bertram [1] showed that for a given $\epsilon>0$ and for almost all integers $n \leqslant x$, as $x \rightarrow \infty, k(G)>|G|^{1-\epsilon}$ for each group $G$ of order $n$.

In his paper of 1963, Brauer asked what the 'true' growth of a lower bound for $k(G)$ in terms of $|G|$ might be. One answer to this was provided by Pyber [14], who proved the lower bound $k(G) \geqslant \epsilon \log _{2}|G| /\left(\log _{2} \log _{2}|G|\right)^{8}$. Experimentally, López and López [9, 10] found that $k(G)>\log _{3}|G|$ if $|G| \leqslant 3^{13}$, and in fact no group has been discovered for which this fails. The groups $G=\operatorname{PSL}(3,4)$ and $G=M_{22}$ both satisfy $k(G)=\left\lceil\log _{3}|G|\right\rceil$.

If we restrict our attention to nilpotent groups (in particular, if we restrict to $|G|$ being a prime power), the ideas of $\mathrm{P}$. Hall immediately give $k(G)>\alpha \log |G|$ for some constant $\alpha$ depending only on $p$, as described in $\S 2$. This was refined by Sherman [16], who showed that if $G$ has nilpotency class $c$, then $k(G)>c\left(|G|^{1 / c}-1\right)+1$. Kovaćs and Leedham-Green [7] produced, for each odd prime $p$, a group $G_{p}$ of order $p^{p}$ with less 
than $p^{3}=\left(\log _{p}\left|G_{p}\right|\right)^{3}$ classes. A natural question, originally formulated by Pyber [14], is whether, for a given $p$, there exists an absolute constant $c$ and a sequence of $p$-groups $\left(G_{n}\right)$, where $G_{n}$ has order $p^{n}$ and $k\left(G_{n}\right)<c n=c \log _{p}\left|G_{n}\right|$.

The aim of this paper is to address what Brauer asked in his 1963 paper by attacking the above question with a computer. We focus on 2-groups, where we can make extensive calculations with the help of the computational software package MAGMA [2].

Let $c_{n}=\min \left\{k\right.$ : there is a group $G$ of order $2^{n}$ with $\left.k(G)=k\right\}$. Our approach consists of three searches. In the first search, we find $c_{n}$ for all $n \leqslant 14$ together with bounds for $c_{15}$. In the second search, we restrict attention to 2-generated 2-groups and find the smallest number of conjugacy classes for such groups of order $2^{15}$ and $2^{16}$. In the third search, we restrict our attention to 2 -groups with coclass at most 3 and with any order.

It appears that $c_{n}$ grows tightly with $n$ except for large occasional jumps. We provide an explanation for this behaviour. The ultimate answer to Brauer's question will depend on a comparison between the frequency and the size of these jumps.

\section{Basic results}

If $|G|=p^{2 m+e}$ with $e=0$ or 1 , then a formula of Hall [13] states

$$
k(G)=m\left(p^{2}-1\right)+p^{e}+r(G)(p-1)\left(p^{2}-1\right)
$$

where $r(G)$ is a non-negative integer. This formula has several implications. First, by noticing that the right-hand side of the equality is at least $m\left(p^{2}-1\right)+p^{e}$, we get that $k(G)>\alpha \log |G|$, where $\alpha$ is a constant depending only on $p$. For $p=2$, we obtain $k(G)=3(m+r(G))+2^{e}$, so that $k(G) \equiv|G|(\bmod 3)$. In fact, Poland showed that if $G$ is a $p$-group such that $r(G)=0$, then $|G| \leqslant p^{p+2}$ and it has coclass 1 . FernándezAlcober and Shepherd [5] recently proved that if $p \geqslant 11$ and $r(G)=0$, then $|G| \leqslant p^{p+1}$. Computational evidence (such as that provided by this paper) suggests that there are bounds on the order and coclass of $p$-groups with a given $r(G)$, which, if true, explains phenomena later in this paper. Also, Poland showed that $k(G)>k(Q)$ and $r(G) \geqslant r(Q)$ if $Q$ is a proper quotient of $p$-group $G$. We summarize the consequences for $c_{n}$. Note that from this point on we consider exclusively 2-groups.

Lemma 2.1. $c_{n} \equiv 1(\bmod 3)$ if $n$ is even, and $c_{n} \equiv 2(\bmod 3)$ if $n$ is odd. Moreover, $c_{n}>c_{n-1}$.

Call a group $G$ of order $2^{n}$ with $k(G)=c_{n}$ a best group. We can establish some properties of sequences of best groups.

Theorem 2.2. Let $G_{n}(n=1,2, \ldots)$ be a sequence of best groups. The coclass of $G_{n}$ grows without bound as $n \rightarrow \infty$.

This follows by combining the following two lemmas.

Lemma 2.3. For each positive integer $c$, there exists a positive real number $\alpha_{c}$, such that if $G$ is a 2-group of coclass $c$, then $k(G) \geqslant \alpha_{c}|G|$. 
Proof. Shalev's proof [12] of the conjectures of Leedham-Green and Newman shows that if $G$ is a 2-group of coclass $c$, then it contains an abelian normal subgroup $A$ of index bounded by a function $f(c)$. This implies that

$$
k(G) \geqslant k(A) /[G: A]=|A| /[G: A]=|G| /[G: A]^{2} \geqslant|G| / f(c)^{2} .
$$

Taking $\alpha_{c}=1 / f(c)^{2}$ gives the result.

Lemma 2.4 (see [6]). Let $H_{n}$ be the Sylow 2-subgroup of $G L\left(n, F_{2}\right)$. The order of $H_{n}$ is $2^{T(n)}$, where $T(n)=(n-1)(n-2) / 2$ and

$$
2^{\left(1 / 12-\epsilon_{n}\right) n^{2}}<k\left(H_{n}\right)<2^{\left(1 / 4+\epsilon_{n}\right) n^{2}}
$$

where $\epsilon_{n} \rightarrow 0$ as $n \rightarrow \infty$.

Proof of Theorem 2.2. Suppose $G_{n}$ is a sequence of best groups whose coclasses form a finite set $C$. Let $\alpha=\min \left\{\alpha_{c} \mid c \in C\right\}$. Then $c_{n} / 2^{n}=k\left(G_{n}\right) /\left|G_{n}\right| \geqslant \alpha>0$ for all $n$. In particular, we have $k\left(H_{n}\right) \geqslant c_{T(n)} \geqslant 2^{T(n)} \alpha$, contradicting Lemma 2.4 for sufficiently large $n$.

\section{The first search; exhaustive for small $n$}

Theorem 3.1. The values of $c_{n}$ for $n \leqslant 14$ are as follows $\left(G_{n}\right.$ a best group):

$\begin{array}{rrcc}n & c_{n} & r\left(G_{n}\right) & \operatorname{coclass}\left(G_{n}\right) \\ 1 & 2 & 0 & 1 \\ 2 & 4 & 0 & 1 \\ 3 & 5 & 0 & 1 \\ 4 & 7 & 0 & 1 \\ 5 & 11 & 1 & 1 \text { or } 2 \\ 6 & 13 & 1 & 2 \\ 7 & 14 & 1 & 2 \\ 8 & 19 & 2 & 2 \\ 9 & 26 & 4 & 3 \text { or } 4 \\ 10 & 28 & 4 & 3 \text { or } 4 \\ 11 & 29 & 4 & 3 \text { or } 4 \\ 12 & 34 & 5 & 3 \text { or } 4 \\ 13 & 35 & 5 & 3 \\ 14 & 37 & 5 & 3\end{array}$

The aim of this search is to compute $c_{n}$ for as many $n$ as possible. We originally used CAYLEY but later checked our results with the quicker system MAGMA. The databases of these systems contain all 2-groups of order $\leqslant 256$, and this allows us immediately to find $c_{n}$ for $n \leqslant 8$. To extend these results, we use the following refinement of Lemma 2.1 . We write $n(G)=\log _{2}(|G|)$. 
Lemma 3.2. If $Q$ is a quotient of the 2-group $G$, then $2 k(G)-3 n(G) \geqslant 2 k(Q)-3 n(Q)$ if $n(G)$ is even, whereas $2 k(G)-3 n(G) \geqslant 2 k(Q)-3 n(Q)-1$ if $n(G)$ is odd.

Proof. If $|G|=2^{2 m+e}$ and $|Q|=2^{2 n+f}$ with $e, f \in\{0,1\}$, then

$$
\begin{aligned}
(2 k(G)-3 n(G))-(2 k(Q)-3 n(Q)) & =6(r(G)-r(Q))+\left(2^{e+1}-3 e\right)-\left(2^{f+1}-3 f\right) \\
& =6(r(G)-r(Q))+f-e
\end{aligned}
$$

Since $r(G) \geqslant r(Q)$, this is non-negative except, possibly, if $f=0$ and $e=1$, in which case it is at least -1 .

The $p$-group generation process of O'Brien [12] creates, for each positive integer $d$, a tree whose vertices are the $d$-generated 2-groups (counted once up to isomorphism). An edge exists from $P$ to $Q$ if $P$ is isomorphic to $Q / \gamma_{c}(Q)$, where $\gamma_{c}(Q)$ is the last non-trivial term of the lower exponent-p central series of $Q$. In that case, we call $Q$ an immediate descendant of $P$. If there is a path from $P$ to $Q$, then we say $Q$ is a descendant of $P$. O'Brien's process allows us to compute immediate descendants (and so descendants) of any given 2-group.

We use Lemma 3.2 and O'Brien's trees to compute $c_{n}$ for increasing $n$. To test, for instance, if there is a group of order $2^{12}$ with $\leqslant 31$ conjugacy classes, we use O'Brien's routine to compute all 2-groups $Q$ with smaller order and $2 k(Q)-3 n(Q) \leqslant 26$. If our group existed, then it would be an immediate descendant of such a $Q$. A computational check shows that no such $Q$ has an immediate descendant of order $2^{12}$ with $\leqslant 31$ conjugacy classes. So $c_{12} \geqslant 34$ and we find all best groups of order $2^{12}$ by using O'Brien's routine to find all groups with $2 k(Q)-3 n(Q) \leqslant 32$ and $|Q| \leqslant 2^{12}$.

This works well until we try to find $c_{15}$. The bound on $2 k(Q)-3 n(Q)$ becomes so large that we have to consider too many groups in O'Brien's trees for this computation to be feasible. The case of 2-generated groups alone (see $\S 4$ ) took a few months to complete. The best we have is that $53 \leqslant c_{15} \leqslant 68$.

We have data on the best groups of order $2^{n}(n \leqslant 14)$ that may be obtained by request from the authors. A few observations are in order. For each $n$ there are 2-generated best groups. For $n=9,10,11,12$, there are also 3 -generated best groups (these being the ones of coclass 4 of those orders). Note that jumps in $c_{n}$ are apparently accompanied by jumps in coclass. The best groups of order $2^{14}$ are extensions of the same point group of order $2^{6}$ by a normal subgroup isomorphic to the direct product $C_{4}^{4}$.

\section{The second search; 2-generated groups}

Since the search for the best groups of order $2^{15}$ ultimately involved too many groups to be feasible, we decided to restrict our attention to 2-generated groups. This permits a lengthy but successful search.

Theorem 4.1. There are 142 2-generated groups of order $2^{15}$ with 68 conjugacy classes. No 2-generated group of order $2^{15}$ has fewer conjugacy classes. There are 92 
2-generated groups of order $2^{16}$ with 70 conjugacy classes. No 2-generated group of order $2^{16}$ has fewer conjugacy classes. Every 2 -generated group of order $2^{17}$ has $\geqslant 74$ conjugacy classes.

This arises by use of the method of the previous section. We inductively construct all 2-generated 2-groups $Q$ with $2 k(Q)-3 n(Q) \leqslant 92$. The largest of these have order $2^{16}$. The groups of order $2^{15}$ are of coclass 3 or 4 . The ones of order $2^{16}$ are of coclass 4 . Note that if $G_{n}$ is one of these groups, then $r\left(G_{n}\right)=15$. We know of no $d$-generated groups, for $d \geqslant 3$, that have the same order as, but fewer conjugacy classes than, the above 2-generated groups.

\section{The third search; groups of coclass $\leqslant 3$}

The paper of Newman and O'Brien [11] presents a method for obtaining all 2-groups of coclass 3 . Since many of our best 2 -groups have coclass $\leqslant 3$, we decided to do an exhaustive study of the number of conjugacy classes of these groups using [11]. All but 1782 sporadic examples naturally fall into 82 families as follows. There are 82 pro- 2 groups, which correspond to the infinite ends of the subtrees of coclass $\leqslant 3$ groups of O'Brien's trees for $1 \leqslant d \leqslant 3$. Mainline groups in family \#i are obtained by taking the exponent- $p$ central quotients of pro-2 group \# $i$. The rest are obtained by taking descendants of these mainline groups. Above a certain vertex (the periodic root), the pattern of descendants is conjecturally periodic. This regularity allows us to find the 2 -groups of coclass 3 with fewest conjugacy classes for all orders $n$. Since the largest of the 1782 sporadic groups has order $2^{14}$, we need not consider them when working with $n>14$. The case of $n \leqslant 14$ was covered in $\S 2$.

For family \#i, for each $i$, we compute the number $f_{i}(n)$ of conjugacy classes of its mainline quotient of order $2^{n}$ for sufficiently large $n$. For instance, family \#2 yields dihedral groups and so $f_{2}(n)=2^{n-2}+3$.

There is a formula for $f_{i}(n)$ of the form $a 2^{n}+$ lower terms ( $a$ independent of $n$ ). For instance, for family \#34, setting $x=2^{[n / 4]}, f_{34}(n)=2^{n-12}+c x^{2}+d x+9$, where the values of $c$ and $d$ depend on $n(\bmod 4)$ as follows: if $n \equiv 0(\bmod 4)$, then $c=27 / 128$ and $d=51 / 16 ;$ if $n \equiv 1(\bmod 4)$, then $c=3 / 8$ and $d=27 / 8 ;$ if $n \equiv 2(\bmod 4)$, then $c=27 / 64$ and $d=33 / 8 ;$ if $n \equiv 3(\bmod 4)$, then $c=3 / 4$ and $d=39 / 8$.

It is easy to see then that no group in family \#2 beats even the mainline groups in family \#34. We carry this method through with all 82 families. It is interesting to observe that $f_{29}(n)=f_{34}(n)+6$, and lengthy computations show that these are the only two families that compete for best coclass 3 groups, in the following sense.

Let $c_{n}^{(3)}=\min \left\{k:\right.$ there is a group $G$ of order $2^{n}$ and coclass $\leqslant 3$ with $\left.k(G)=k\right\}$. If the group $G$ has order $2^{n}$, coclass $\leqslant 3$, and $k(G)=c_{n}^{(3)}$, then $G$ will be called a best coclass 3 group. 
Theorem 5.1. For $n \leqslant 14, c_{n}^{(3)}$ is given by Theorem 3.1, and, for $15 \leqslant n \leqslant 26$, the values of $c_{n}^{(3)}$ are given by

$\begin{array}{rr}n & c_{n}^{(3)} \\ 15 & 68 \\ 16 & 76 \\ 17 & 110 \\ 18 & 148 \\ 19 & 242 \\ 20 & 373 \\ 21 & 617 \\ 22 & 1123 \\ 23 & 1493 \\ 24 & 4993 \\ 25 & 6341 \\ 26 & 11911\end{array}$

The best groups of coclass 3 of order $2^{15}$ are located in families \#30, \#32, \#35 and \#42. The best of order $2^{16}$ are in family \#42 and of orders $2^{17}, 2^{18}, 2^{20}$ and $2^{21}$ in family \#20. As for $n=19$ and $n \geqslant 22$, the following claim is verified for $n \leqslant 26$ and is expected to hold in general. (A rigorous check of it would be far too lengthy; even the computational evidence for it takes several weeks to obtain.)

Claim 5.2. Suppose $n=19$ or $n \geqslant 22$. The best coclass 3 groups of order $2^{n}$ depend on $n(\bmod 4)$ as follows.

(i) If $n \equiv 1(\bmod 4)$, then they are descendants of the mainline group of order $2^{n-2}$ of family \#34.

(ii) If $n \equiv 2(\bmod 4)$, then they are descendants of the mainline group of order $2^{n-3}$ of family \#29.

(iii) If $n \equiv 3(\bmod 4)$, then they are descendants of the mainline group of order $2^{n-4}$ of family \#29.

(iv) If $n \equiv 0(\bmod 4)$, then they are descendants of the mainline group of order $2^{n-5}$ of family \#34.

In each of the four cases, there is a formula for $c_{n}^{(3)}$ of the form $c_{n}^{(3)}=2^{n-13}+b x^{3}+$ $c x^{2}+d x+e$, where $x=2^{[n / 4]}$ and where $b, c, d$ and $e$ are rational numbers depending only on the congruence class of $n((\bmod 4))$. For instance, it appears that for $n \equiv 1$ $(\bmod 4), b=107 / 14336, c=-11 / 256, d=119 / 16$ and $e=-81 / 7$. 
Acknowledgements. N.B. was partly supported by the Sloan Foundation and NSF grant DMS 96-22590. He thanks God for leading him to results. J.L.W. was partly supported by the Henry Luce Foundation and NSF grant DMS 97-09388. Both authors thank Eamonn O'Brien for his generous software help.

\section{References}

1. E. A. Bertram, On large cyclic subgroups of finite groups, Proc. Am. Math. Soc. 56 (1976), 63-66.

2. W. Bosma AND J. J. CANnON, Handbook of Magma functions (School of Mathematics and Statistics, University of Sydney, 1996).

3. R. BRAUER, Representations of finite groups. Lectures on Modern Mathematics (ed. T. L. Saaty), vol. I (Wiley, New York, 1963).

4. P. ERdös AND P. TURÁn, On some problems of a statistical group theory, IV, Acta Math. Acad. Sci. Hung. 19 (1968), 413-435.

5. G. A. Fernández-Alcober and R. T. Shepherd, On the order of $p$-groups of abundance zero, J. Algebra 201 (1998), 392-400.

6. G. Higman, Enumerating p-groups, I, Inequalities, Proc. Lond. Math. Soc. (3) 10 (1960), 24-30.

7. L. G. Kovaćs AND C. R. LeEdham-GREen, Some normally monomial p-groups of maximal class and large derived length, Q. J. Math. Oxford 37 (1986), 49-54.

8. E. LANDAU, Klassenzahl binärer quadratischer Formen von negativer Discriminante, Math. Ann. 56 (1903), 674-678.

9. A. V. LÓPEZ AND J. V. LÓPEZ, Classification of finite groups according to the number of conjugacy classes, Isr. J. Math. 51 (1985), 305-338.

10. A. V. LóPEz AND J. V. LóPEz, Classification of finite groups according to the number of conjugacy classes, II, Isr. J. Math. 56 (1986), 188-221.

11. M. F. Newman and E. A. O'Brien, Classifying 2-groups by coclass, Trans. Am. Math. Soc. 351 (1999), 131-169.

12. E. A. O'BRIEN, The p-group generation algorithm, J. Symbolic Comput. 9 (1990), 677698.

13. J. Poland, Two problems on finite groups with $k$ conjugate classes, J. Aust. Math. Soc. 8 (1968), 49-55.

14. L. PyBER, Finite groups have many conjugacy classes, J. Lond. Math. Soc. (2) 46 (1992), 239-249.

15. A. Shalev, The structure of finite $p$-groups: effective proof of the coclass conjectures, Invent. Math. 115 (1994), 315-345.

16. G. J. SHERMAN, A lower bound for the number of conjugacy classes in a finite nilpotent group, Pac. J. Math. 80 (1979), 253-254. 